[I065] Information provided by manufacturers on equipment (BSS ART 78)

Caterina Brusasco*

Cocir - Radiation Protection Fg Chair, Iba R\&d Compliance Expert, Louvain La Neuve, Belgium

* Corresponding author.

The BSS article 78 requires manufacturers to provide to undertakings information that can be used by them to perform their own risk evaluation. Unfortunately, it does not specify which information and which format, with a risk of diverging national interpretations and requirements in EU. Medical devices are regulated by the MDD Directive for their placing on the EU market and manufacturers applies the related relevant standards to ensure safety of their equipment. The MDD Directive and the related standards already require manufacturers to inform users of any residual risk, including radiological ones, in the instruction for use and the accompanying documentation. Manufactures are open to cooperate with regulators and professional societies to ensure useful information is provided to undertakings in the framework of the BSS regulation, limiting diverging national interpretations and practices.

Purpose. To provide a description of the information on radiationrelated risks that is already provided to users in the Instructions for Use and accompanying documentation and to provide a base of discussion for users on how such information covers all possible needs as required by EU legislation and International standards.

https://doi.org/10.1016/j.ejmp.2018.06.137

\section{[1066] EFOMP guidelines on the transposition of EU BSS art.60 in} national legislations

Alberto Torresin ${ }^{\mathrm{a},{ }^{*}}$, John Damilakis ${ }^{\mathrm{b}}$, Manuel Bardies $^{\mathrm{c}}$, Marco Brambilla ${ }^{\mathrm{d}}$, Annalisa Trianni ${ }^{\mathrm{e}}$, Stephen Evans ${ }^{\mathrm{f}}$, Ana Rita Figueira ${ }^{g}$, Virginia Tsapaki ${ }^{\text {h }}$

a Asst Grande Ospedale Metropolitano Niguarda Hospital, Department of Medical Physics, Milan, Italy

${ }^{\mathrm{b}}$ University of Crete, Medical Physics, Iraklion, Greece

'Centre de Recherches En Cancérologie de Toulouse, Université Toulouse III - Paul Sabatier, Inserm, Umr1037 Crct, Toulouse, France

dUniversity Hospital "Maggiore Della Carità", Medical Physics, Novara, Italy

${ }^{\mathrm{e}}$ Udine University Hospital, Medical Physics, Udine, Italy

${ }^{\mathrm{f}}$ Northampton, UK, Retired, Formerly Head of Medical Physics, Northampton, United Kingdom

${ }^{g}$ Centro Hospitalar de São João, Radiotherapy Department, Porto, Portugal

${ }^{\mathrm{h}}$ Konstantopoulio General Hospital, Athens, Greece, Medical Physics, Nea Ionia, Greece

* Corresponding author.

Purpose. The aim of EFOMP's document is to clarify the position on article 60.3 (c-f) of the EU BSS Directive regarding optimization processes.

Results. The equipment used for interventional radiology and computed tomography and any new equipment used for planning, guiding and verification purposes, shall be able to inform the practitioner (radiologist, oncologist, technologist etc.) about the relevant parameters for assessing the quantity of radiation produced (angles, distances, exposure parameters, etc.). Furthermore, article 60.3(e) states that equipment used for interventional radiology and com- puted tomography must have the capacity to transfer this information to the record of the examination.

The most common parameters used to quantify the radiation output will be discussed taking into account their impact on patient dosimetry.

The Medical Physics Expert (MPE) role will be presented and discussed in:

1) Measuring the output of the radiological modality, estimating the related uncertainty, periodically verifying the calibration

2) Planning optimization strategies, dosimetric evaluations and quality controls

3) Comparing different modalities/equipment/protocols/exams with the purpose of optimizing the procedures. In this case, it might be necessary to translate the output metrics to effective dose and/or organ doses in order to compare doses between different modalities for optimization purposes.

It is very important that the MPE defines the correct information to be captured that is related to the patient radiation dose.

Some radiological metrics are not simple to understand (CTDI, SSDE for examples) and the MPE has to guide the medical community in their use.

Conclusions. EFOMP also recommends that all medical equipment should document the maximum uncertainty of the dosimetric index displayed. The uncertainties depend on the acquisition parameters. It is important that the manufacturers specify the maximum uncertainty of the dosimetric index.

https://doi.org/10.1016/j.ejmp.2018.06.138

[1067]

Abstract not available

\section{[1068] Radiation effects on the embryo and fetus} Cari Borrás*

Aapm, Chair, International Educational Activities Committee, Washington DC, United States

* Corresponding author.

The effects of in utero radiation should not be confused with heritable effects that can manifest by irradiating germ cells (oocytes or spermatocytes) before conception, causing chromosomal aberrations and/or mutations in the genes themselves. These (stochastic) genetic effects have been documented in animals and insects, but there is no epidemiological evidence of excess heritable disease in the offspring of humans irradiated before conception. The irradiation of somatic cells of the embryo/fetus can cause both tissue injuries and stochastic effects. Tissue injuries are dependent on the fetal stage of development and have been documented in irradiated cohorts of Hiroshima/Nagasaki atomic bomb survivors and residents around Chernobyl and the Mayak Plant/Techa River in the Southern Urals, as well as after medical radiation. According to the 2013 US National Council on Radiation Protection and Measurements Report 174, embryo lethality can occur in the pre-implantation period at doses above $100 \mathrm{mGy}$; malformations (such as microcephaly, micrognathia and cleft palate) may occur during organogenesis above $100 \mathrm{mGy}$, and severe mental retardation, in the weeks $8-25^{\text {th }}$ post conception, with a dose threshold of $300 \mathrm{mGy}$. The most sensitive period is the $8-15^{\text {th }}$ week, where a decrease of up to 30 IQ units has been observed. As for radiation-induced stochastic effects, although cancer has been 